\title{
Electron-Electron Bremsstrahlung Emission and the Inference of Electron Flux Spectra in Solar Flares
}

\author{
Eduard P. Kontar ${ }^{1}$, A. Gordon Emslie ${ }^{2}$, Anna Maria Massone ${ }^{3}$, Michele Piana ${ }^{4}$, John C. \\ Brown $^{1}$, and Marco Prato ${ }^{5}$
}

\begin{abstract}
Although both electron-ion and electron-electron bremsstrahlung contribute to the hard Xray emission from solar flares, the latter is normally ignored. Such an omission is not justified at electron (and photon) energies above $\sim 300 \mathrm{keV}$, and inclusion of the additional electron-electron bremsstrahlung in general makes the electron spectrum required to produce a given hard X-ray spectrum steeper at high energies.

Unlike electron-ion bremsstrahlung, electron-electron bremsstrahlung cannot produce photons of all energies up to the maximum electron energy involved. The maximum possible photon energy depends on the angle between the direction of the emitting electron and the emitted photon, and this suggests a diagnostic for an upper cutoff energy and/or for the degree of beaming of the accelerated electrons.

We analyze the large event of January 17, 2005 and show that the upward break around $400 \mathrm{keV}$ in the observed hard X-ray spectrum is naturally accounted for by the inclusion of electron-electron bremsstrahlung. Indeed, the mean source electron spectrum recovered through a regularized inversion of the hard X-ray spectrum, using a cross-section that includes both electron-ion and electron-electron terms, has a relatively constant spectral index $\delta$ over the range from electron kinetic energy $E=200 \mathrm{keV}$ to $E=1 \mathrm{MeV}$. However, the level of detail discernible in the recovered electron spectrum is not sufficient to determine whether or not any upper cutoff energy exists.
\end{abstract}

Subject headings: processes: radiation; Sun: flares; Sun: X-rays

\section{Introduction}

The spatially integrated hard X-ray spectrum $I(\epsilon)$ (photons $\mathrm{cm}^{-2} \mathrm{~s}^{-1} \mathrm{keV}^{-1}$ at the Earth) is produced by bremsstrahlung of accelerated elec-

\footnotetext{
${ }^{1}$ Department of Physics \& Astronomy, University of Glasgow G12 8QQ, UK

eduard@astro.gla.ac.uk, john@astro.gla.ac.uk

${ }^{2}$ Department of Physics, Oklahoma State University, Stillwater, OK 74078

gordon.emslie@okstate.edu

${ }^{3}$ CNR-INFM LAMIA, via Dodecaneso 33, I-16146 Genova, Italy(massone@ge.infm.it)

${ }^{4}$ Dipartimento di Informatica, Università di Verona, Ca`Vignal 2, Strada le Grazie 15, I-37134 Verona, Italy (michele.piana@univr.it)

${ }^{5}$ Dipartimento di Matematica, Università di Modena e Reggio Emilia, via Campi 213/b, I-41100 Modena, Italy (marco.prato@unimo.it)
}

trons, characterized (Brown, Emslie, \& Kontar 2003 ) by a mean electron flux spectrum $\bar{F}(E)$ (electrons $\mathrm{cm}^{-2} \mathrm{~s}^{-1} \mathrm{keV}^{-1}$ at the Sun) and related to $I(\epsilon)$ through

$$
I(\epsilon)=\frac{1}{4 \pi R^{2}} \bar{n} V \int_{\epsilon}^{\infty} \bar{F}(E) Q(\epsilon, E) d E,
$$

where $Q(\epsilon, E)$ is the bremsstrahlung cross-section $\left(\mathrm{cm}^{2} \mathrm{keV}^{-1}\right)$ differential in photon energy, $R=$ $1 \mathrm{AU}$, and the mean target density $\bar{n}\left(\mathrm{~cm}^{-3}\right)$ is defined by $\bar{n}=V^{-1} \int n(\mathbf{r}) d V$. Bremsstrahlung in the energy range $\gtrsim 10 \mathrm{keV}$ is produced by energetic electrons scattering off both protons/ions and electrons (both free and bound in atoms); these contributions are summed to give the total differential bremsstrahlung cross-section $Q(\epsilon, E)$. 
For electron energies $\lesssim 300 \mathrm{keV}$, the contribution from electron-electron bremsstrahlung can be safely ignored (Haug 1975). However, for higher energies this is no longer the case. Generally, for a given electron spectrum, the additional electron-electron bremsstrahlung yield acts to flatten (harden) the photon spectrum in this energy range (see, e.g., Haug 1975). Equivalently, the inclusion of electron-electron bremsstrahlung requires, for a given photon yield, a softer (steeper) electron spectrum than would be required assuming electron-ion bremsstrahlung alone.

The Ramaty High Energy Solar Spectroscopic Imager (RHESSI) has opened a new era in the study of hard X-ray spectra from solar flares. With the high-resolution hard X-ray spectra made available by RHESSI, an investigation into the form of the hard X-ray spectrum above $\sim 300 \mathrm{keV}$ with the full (electron-ion + electron-electron) cross-section is now warranted.

In this paper we therefore study the effect of adding the electron-electron bremsstrahlung term on the mean electron spectrum corresponding to a given hard X-ray spectrum. In $\S 2$ we discuss the forms of the electron-ion and electron-electron cross-sections, and we show that the presence of an upper limit to the photon energy in the electronelectron process can in principle provide evidence for a high-energy cutoff in the electron spectrum and/or evidence of strong anisotropy in the injected electron distribution. In $\S 3$ we discuss the sample event (on January 17, 2005) chosen for analysis. In $\S 4$ we present the form of the electron spectrum corresponding to the observed photon spectrum, using both forward-fitting (e.g, Holman et al. 2003) and regularization (Piana et al. 2003) techniques in conjunction with the full (electron-ion + electron-electron) bremsstrahlung cross-section. In $\S 5$ we discuss the results obtained, and in particular we point out that certain features in the electron spectrum inferred using the electron-ion bremsstrahlung cross-section are artifacts that can vanish when the full, correct, cross-section is employed.

\section{Form of the Bremsstrahlung Cross- Section}

The cross-section (e.g., Koch \& Motz 1959) for electron-ion bremsstrahlung scales as $Z^{2}$, where
$Z$ is the atomic number of the ion. Further, in consideration of electron-electron bremsstrahlung, the possible binding of target electrons to their host ions in a neutral or partially-ionized medium is not significant (E. Haug, personal communication). Hence, in a quasi-neutral target of particles with atomic number $Z$, the bremsstrahlung crosssection per atom for emission of a photon of energy $\epsilon$ by an electron of energy $E$ is in general equal to

$$
Q(\epsilon, E)=Z^{2} Q_{e-p}(\epsilon, E)+Z Q_{e-e}(\epsilon, E) .
$$

were $Q_{e-p}(\epsilon, E)$ and $Q_{e-e}(\epsilon, E)$ are the crosssections for bremsstrahlung in electron-proton, and electron-electron collisions, respectively.

The form of $Q_{e-e}(\epsilon, E)$, averaged over solid angle in the rest frame of the target electron has been given 11 by Haug (1998), while the solid-angleaveraged form in the zero-momentum ("center-of mass") frame has been given by Haug (1989). Neither of these formulae are strictly appropriate to the case of an electron beam incident on a warm plasma: the target electrons, unlike the ions, have a velocity that may be comparable to the velocity of the electrons in the impinging beam, so that a range of injected particle/target particle relative velocities exist for a given injected electron energy. However, as verified through numerical simulation, for low electron energies (from $\sim 10$ to $\sim 200 \mathrm{keV}$ ), the form of $\bar{F}(E)$ corresponding to a given hard X-ray spectrum, inferred using the cross-section (2), does not differ, within statistical uncertainties in the photon flux, from the form of $\bar{F}(E)$ obtained using the electron-ion cross-section alone. Hence, only at electron energies $\gtrsim 200 \mathrm{keV}$ is the inclusion of the electronelectron bremsstrahlung term necessary, and, for such energies the velocity of the target particles is relatively insignificant. The target particle rest frame is, therefore, a better approximation to the observer frame than is the zero-momentum frame. Hence, use of the electron-electron cross-section in the target particle rest frame is more appropriate.

When the maximum electron energy is much larger than the photon energies under consideration, the photon spectrum resulting from

\footnotetext{
${ }^{1}$ Note that in the formula for $H(\epsilon, k, x)$ for the case $k>\frac{1}{2}$ (page 347 of that paper), the term $(\epsilon r / x+s)$ on line 3 of the equation should be replaced by $(\epsilon r / w+s)-$ E. Haug, personal communication.
} 
a power-law spectrum of electrons $\bar{F}(E) \sim$ $E^{-\delta}$ is also close to power-law form $I(\epsilon) \sim$ $\epsilon^{-\gamma}$ (Haug 1989). However, while for pure electron-ion bremsstrahlung $\gamma \simeq \delta+1$, for pure electron-electron bremsstrahlung a significantly shallower photon spectrum, with $\gamma \simeq \delta$, results. Thus, the importance of the electron-electron bremsstrahlung contribution increases with photon energy and the enhanced emission per electron leads to a flattening of the photon spectrum $I(\epsilon)$ produced by a given $\bar{F}(E)$, or, equivalently, a steepening of the $\bar{F}(E)$ form required to produce a given $I(\epsilon)$.

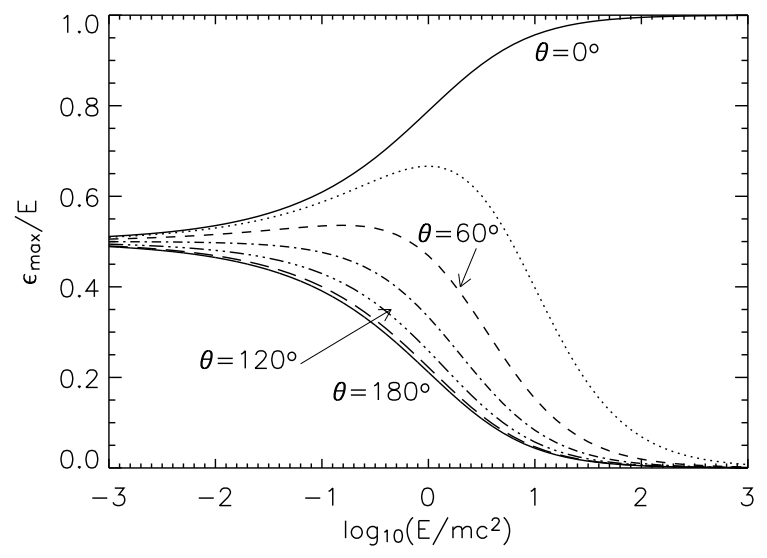

Fig. 1.- Maximum photon energy $\epsilon_{\max }$ produced by electron-electron bremsstrahlung, expressed as a fraction of the incident electron energy $E$ (in units of the electron rest mass $m c^{2}$ ), for various values of $\theta$, the angle between the incoming electron and the outgoing photon trajectories For clarity, only curves for $\theta=0^{\circ}, 60^{\circ}, 120^{\circ}$, and $180^{\circ}$ are labelled; the curves for $\theta=30^{\circ}, 90^{\circ}$ and $150^{\circ}$ lie between these.

It is also important to note that while the electron-ion cross-section is finite for all $\epsilon<E$, the "laboratory frame" cross-section for electronelectron bremsstrahlung vanishes above a maximum photon energy, due to the necessarily finite energy carried by the recoiling target electron. Quantitatively (Haug 1975),

$$
\epsilon_{\max }=\frac{E}{E+2-\sqrt{E(E+2)} \cos \theta},
$$

where $E$ is the electron kinetic energy in the laboratory frame (in units of the electron rest energy $m c^{2}$ ) and $\theta$ is the angle between the incom- ing electron and the outgoing photon trajectories. For highly non-relativistic electrons $(E \ll 1)$, $\epsilon_{\max } \rightarrow E / 2$ for all values of $\theta$. Only for highly relativistic electrons $(E \gg 1)$ and $\theta=0$ (a singular case) does $\epsilon_{\max } \rightarrow E$; for all other situations $\epsilon_{\max }$ is less than $E$ and approaches 0 as $E \rightarrow \infty$ (Figure 1).

This result has important implications for the form of the photon spectrum produced by electron-electron bremsstrahlung. If the electron spectrum $\bar{F}(E)$ has a maximum energy $E_{\max }$ that is not highly relativistic, then while electron-ion bremsstrahlung will generate photons at all energies up to $E_{\max }$, electron-electron bremsstrahlung will produce no photons at all in the range $\epsilon_{\max }<\epsilon \leq E_{\max }$. The entire spectrum above $\epsilon_{\max }$ will therefore be produced completely by electron-ion bremsstrahlung; the flattening of the photon spectrum associated with the electronelectron contribution disappears and the relationship between $I(\epsilon)$ and $\bar{F}(E)$ reverts to the form appropriate to electron-ion bremsstrahlung alone.
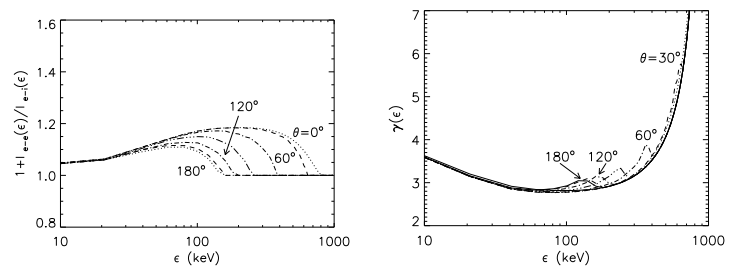

Fig. 2.- Left panel: Ratio of the total photon spectrum to that produced by electron-ion bremsstrahlung alone, for four different viewing angles $\theta$. The mean source electron spectrum is a power law with spectral index $\delta=2$ and an upper cutoff energy at $1 \mathrm{MeV}$; Right panel: Corresponding local spectral indices $\gamma(\epsilon)=-d \log I(\epsilon) / d \log \epsilon$. Note the sharp features in $\gamma$ caused by the absence of electron-electron emission above a certain photon energy $\epsilon_{\max }$ (see Figure 11). For clarity, only select curves have been labelled in both panels.

Note also that the maximum photon energy $\epsilon_{\max }$ depends significantly on the viewing angle $\theta$. Hence, if the injected electron distribution is highly beamed, the strong angular dependence of the maximum photon energy produced permits a determination of the direction of the beam. For example, for $E_{\max }=1 \mathrm{MeV}$ and $\theta=90^{\circ}$, there should be evidence for such a high-energy cutoff in 
the photon spectrum around $250 \mathrm{keV}$ (Figure 1). Figure 2 shows the effect of such a $1 \mathrm{MeV}$ upper energy cutoff on the total (electron-ion + electronelectron) photon spectrum $I(\epsilon)$ and on its local spectral index $\gamma=-d \log I(\epsilon) / d \log \epsilon$. There is an abrupt step in $\gamma$ at $\sim 250 \mathrm{keV}$; this step moves towards larger energies as $\theta$ is reduced, so that the inferred value of $E_{\max }$ depends on the value of $\theta$ appropriate.

\section{Data analysis}

In selecting suitable events for analysis, we searched for a clear identification of high-energy photons in the flare light curve, and specifically a count rate high enough to provide good count statistics in energy channels above $300 \mathrm{keV}$. Quasilogarithmic energy binning was used in order to enhance the signal-to-noise ratio in each energy channel and the time bins were chosen equal to RHESSI's rotation period (as given for the time of the flare) to ensure that there is no differential modulation of the light curve from varying aspects of the imaging grids.

The data were corrected for the following effects: decimation, detector energy response, detector livetime, attenuator transmission, imaging grid transmission, and pulse pile-up. These steps were performed using standard software incorporating the most up to date information on the instrumental calibration (Schwartz et al. 2002). The background was then subtracted by using the SPEX package to interpolate between two background time intervals, one before and one after the flare. Data from detectors 2 or 7 were not used, because their energy resolution is significantly poorer than for the other detectors (Smith et al. 2002).

Figure 3 shows the photon spectrum for the time interval 09:43:16 - 09:44:24 UT (the time of approximate peak flux) for the 2005 January 17 (GOES class X3.8) event. This event, which produced several strong gamma-ray lines, was previously studied by Kontar \& Brown (2006), who concluded that the pitch angle distribution for electrons up to $\sim 300 \mathrm{keV}$ is close to isotropic. We focus attention on the highest energy spectrum $(\epsilon>200 \mathrm{keV})$ in this paper.

This event was located at position $(x=$ $380^{\prime \prime}, y=320^{\prime \prime}$ ) on the solar disk, corresponding to a heliocentric angle $\sim 30^{\circ}$. Consequently,

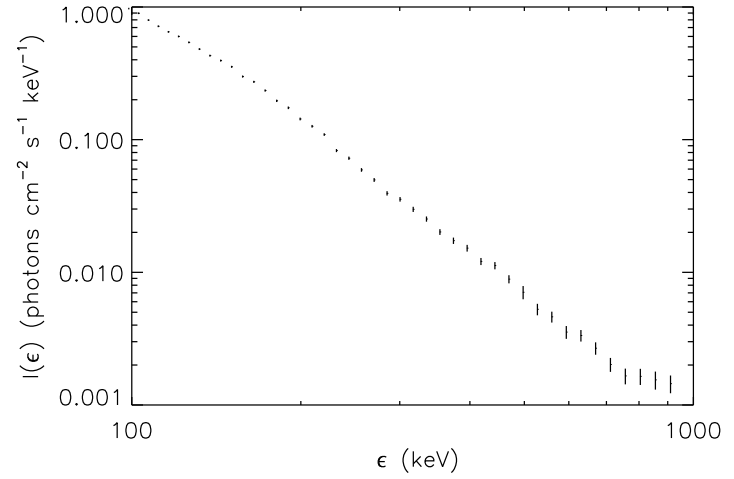

Fig. 3.- Photon spectrum for the time interval 09:43:16 - 09:44:24 UT in the 2005 January 17 event with gamma-lines removed.

the assumption of a downward-directed electron beam leads to angles $\theta$ between the beam direction and the observer in the second quadrant; this enhances the possibility of observing the spectral features noted in $\S 2$ associated with the upper limit to electron-electron bremsstrahlung emission (see Figures 1 and 2).

\section{Determination of the Mean Source Electron Spectrum}

Before attempting to determine the form of the mean source electron spectrum responsible for the observed hard X-ray/gamma-ray continuum, it is first necessary to subtract the emission from strong gamma-ray spectral lines. In the energy range under consideration, the two most significant ranges for which this subtraction is necessary are (483-512) keV and (829-882) keV. The corrected spectrum is presented in Figure 3. The first of these corresponds to the electron-positron annihilation line at $511 \mathrm{keV}$ and its associated positronium continuum at lower energies, the second to a variety of strong emission lines from ${ }^{27} \mathrm{Al},{ }^{54} \mathrm{Cr}$ and ${ }^{56} \mathrm{Fe}$ (see Table 1 in Ramaty, Kozlovsky, \& Lingenfelter 1979; Table 1 in Kozlovsky, Murphy, \& Ramaty 2002). These lines were "removed" by replacing the data in these ranges with a smooth interpolation of the continuum spectrum on either side of each feature.

The residual photon spectra then represent principally bremsstrahlung continuum, with an 
emissivity given by equation (11). These continuum spectra were then used to determine the mean electron flux spectrum $\bar{F}(E)$ in the source, using two different, well-established, methodologies for solution of equation (1).

\subsection{Forward Fit}

Here we follow the procedure of Holman et al. (2003) and assume that the mean electron spectrum is the sum of a low-energy Maxwellian, plus a broken power law of the form

$$
\bar{F}(E)=\left\{\begin{array}{l}
A E^{-\delta_{1}} ; \quad E<E_{\mathrm{brk}} \\
A E_{\mathrm{brk}}^{\delta_{2}-\delta_{1}} E^{-\delta_{2}} ; \quad E \geq E_{\mathrm{brk}}
\end{array}\right.
$$

Because the Maxwellian part of $\bar{F}(E)$ (with a characteristic temperature $T \simeq 3 \mathrm{keV}$ ) is utterly insignificant at energies $E>200 \mathrm{keV}$, it is not necessary to consider this component in our analysis.

Calculation, using equation (10), of the photon spectrum for an $\bar{F}(E)$ of the form (44), and comparison with the observed $I(\epsilon)$ above $\epsilon=200 \mathrm{keV}$, permits determination of the bestfit values of the four parameters $\left(A, E_{\mathrm{brk}}, \delta_{1}, \delta_{2}\right)$. We performed such a forward fit for two forms of the bremsstrahlung cross-section: $Q_{\mathrm{e}-\mathrm{i}}(\epsilon, E)=$ $Z^{2} Q_{e-p}(\epsilon, E)$ (which includes electron-ion bremsstrahlung only) and $Q_{\text {tot }}(\epsilon, E)=Z^{2} Q_{e-p}(\epsilon, E)+Z Q_{e-e}(\epsilon, E)$ (which includes both electron-ion and electronelectron bremsstrahlung). Mean values $\langle Z\rangle=$ 1.2 and $\left\langle Z^{2}>=1.44\right.$ (representative of mean solar abundances) were assumed.

Using the cross-section $Q_{\mathrm{e}-\mathrm{i}}(\epsilon, E)$, representing only electron-ion bremsstrahlung, results in best-fit values $\delta_{1}=3.4, \delta_{2}=2.9$, and $E_{\mathrm{brk}}=$ $445 \mathrm{keV}$. Using the more correct cross-section $Q_{\text {tot }}(\epsilon, E)$ (which incorporates both electron-ion and electron-electron terms) gives $\delta_{1}=3.5, \delta_{2}=$ 3.1 , and $E_{\mathrm{brk}}=431 \mathrm{keV}$. The forms of both of these fits are shown in Figure 4. While inclusion of the electron-electron bremsstrahlung term results in little change to the form of $\bar{F}(E)$ at low energies, its inclusion does lead to the break energy moving downward from $E \sim 450 \mathrm{keV}$ to $E \sim 430 \mathrm{keV}$, and to the spectral index for the high-energy component steepening from $\delta \simeq 2.9$ to $\delta \simeq 3.1(\Delta \delta \simeq 0.2)$. Such a steepening of $\bar{F}(E)$, and the energy above which it becomes significant, are in accordance with the expectations expressed

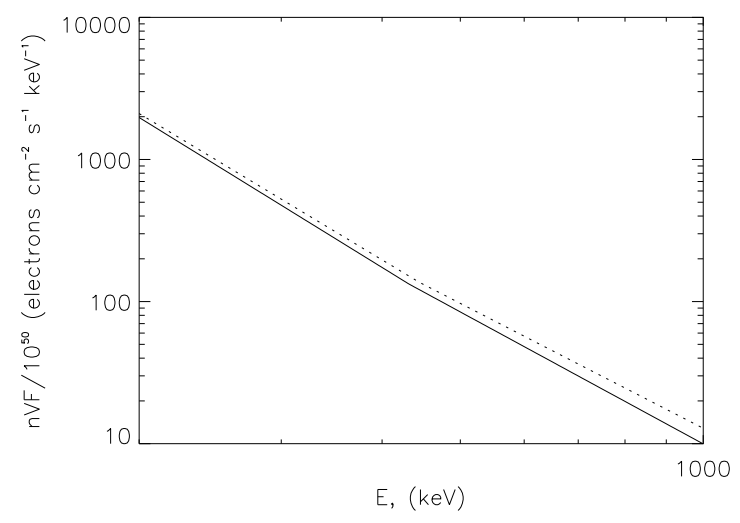

Fig. 4.- Forward-fit forms of $\bar{n} V \bar{F}(E)$ for the selected flare. The dashed curve assumes electronion bremsstrahlung only; the solid curve includes the additional electron-electron term.

in $\S 1$ and with earlier quantitative estimates based on hardening of hard X-ray spectra (e.g., Vestrand 1988).

\subsection{Regularized Inversion}

Piana et al. (2003) have demonstrated the construction of smooth, regularized, forms for the mean electron flux spectrum $\bar{F}(E)$ from highresolution RHESSI photon spectra $I(\epsilon)$. The advantage of this method is that it is not necessary to assume an empirical form for the spectrum. Additionally, as shown by Brown et al. (2006), this method is capable of revealing accurately the overall "shape" of the electron spectrum and indicating the presence and approximate form of smallscale features of sufficient amplitude, if present.

Figure 5 shows the recovered $\bar{F}(E)$ solution for the same photon spectrum used in the forward fit procedure of Figure 4. The results are presented in the form of a confidence strip, a set of different realizations of $\bar{F}(E)$, each curve corresponding to a different realization of the noisy data set $I(\epsilon)$.

It is clear that the $\bar{F}(E)$ recovered using the full cross-section (2), including electron-electron bremsstrahlung, is, for $E \gtrsim 300 \mathrm{keV}$, steeper (spectral index greater by $\sim 0.4$ ) than the $\bar{F}(E)$ recovered assuming purely electron-ion emission. This result is consistent not only with the forward-fit results of the previous subsection but also with the physical expectations enunciated in $\S 1$. More- 


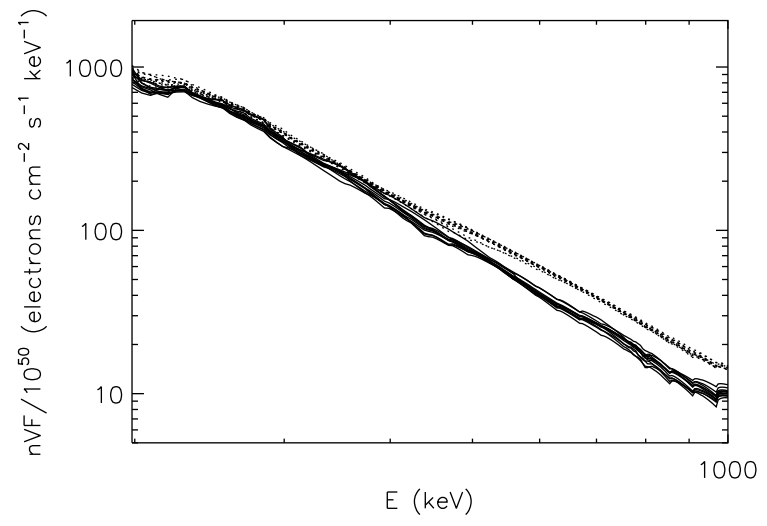

Fig. 5.- Recovered forms of the quantity $\bar{n} V \bar{F}(E) \quad$ (in units of $10^{50}$ electrons $\mathrm{cm}^{-2} \mathrm{~s}^{-1} \mathrm{keV}^{-1}$; see equation 11) using a zero-order regularization technique and presented as a "confidence strip," i.e., a series of solutions, each based on a realization of the data consistent with the size of the uncertainties. The dashed lines assume electron-ion emission only; the solid lines include the additional electron-electron emission term.

over, the dashed confidence strip (corresponding to use of the electron-ion cross-section alone) has an upward break near $E=400 \mathrm{keV}$ (as can be verified visually by looking along - rather than at the strip). However, the true form of $\bar{F}(E)$, as exhibited by the solid confidence strip, has a rather featureless power-law form over the energy range from 200 - $1000 \mathrm{keV}$. Consequently, use of the full cross-section, including the electron-electron term that becomes important at energies $\gtrsim 300 \mathrm{keV}$, removes the need to account for the $\sim 400 \mathrm{keV}$ energy that characterizes the (unphysical) upward break in $\bar{F}(E)$ that appears when only the partial (electron-ion) cross-section is used in the analysis.

\section{Discussion and Conclusions}

As expected, recognition of the growing importance of electron-electron bremsstrahlung at high energies reduces, for a given hard X-ray spectrum, the number of high-energy electrons required to produce it; this leads to a steepening in the inferred mean source electron spectrum $\bar{F}(E)$ above $\sim 400 \mathrm{keV}$. For the January 17, 2005 event studied, use of the electron-ion cross-section alone leads, whether by forward fitting or regularized inversion, to the inference of an upward break $(\Delta \gamma \simeq 0.4)$ in $\bar{F}(E)$ at $E \simeq 400 \mathrm{keV}$ (Figure [5) However, when both electron-ion and electronelectron bremsstrahlung emission are considered, this break disappears, resulting in an $\bar{F}(E)$ that has a straightforward power-law form over the energy range from 100 - $1000 \mathrm{keV}$. Single-power law suggests electron acceleration without characteristic energy and corresponding characteristic scale. Careful interpretation is therefore necessary when faced with apparent hard X-ray spectral changes in this energy range.

One process that can, for a sufficiently strong magnetic field, operate strongly in the few 100 $\mathrm{keV}$ range and so affect this argument is gyrosynchrotron emission. However, the presence of this additional emission mechanism would cause the $\bar{F}(E)$ to bend downward at higher energies. The fact that, after inclusion of the electron-electron contribution, $\bar{F}(E)$ has no such bend puts an upper limit on the importance of gyrosynchrotron emission and so an upper limit $(\sim 10 \mathrm{kG})$ on the strength of the ambient magnetic field.

Trottet et al. (1998) report very significant upward breaks $\left(\Delta \gamma \simeq 1.2-2 ; E_{\mathrm{brk}} \simeq 400 \mathrm{keV}\right)$ in the hard X-ray spectrum for a series of intervals during an electron-dominated gamma-ray event on 1990 June 11. We agree with these authors that the inclusion of electron-electron bremsstrahlung cannot account for such breaks. However, Vestrand (1988) reports that "most flares show a break $\simeq 0.5$ " occurring at an energy " $\simeq 300-400 \mathrm{keV"}$ and a similar statement is made by Dennis (1985) (however, he also reports a much larger spectral break $[\Delta \gamma \simeq 2]$ in an event observed on June 4, 1980). Such modest $(\Delta \gamma \simeq 0.5)$ upward breaks at a photon energies $\epsilon \simeq 300-400 \mathrm{keV}$ are naturally accounted for by including the contribution from electron-electron bremsstrahlung; other considerations, such as energy-dependent anisotropy (Li 1995) or a separate emission/acceleration process (e.g., Heristchi 1986) are in general not required.

Only features common to all (or at least nearly all) realizations of $\bar{F}(E)$ can be considered real. Using this criterion, one must concede that the recovered confidence strip (Figure 5) is sufficiently wide that no firm evidence for a sudden change in the local spectral index $\gamma$ (cf. Figure 2) can be claimed. Hence the data do not provide compelling evidence for either strong beaming of the 
accelerated electrons or an upper-energy cutoff $E_{\max }$ in the accelerated electron energy distribution. Such an assessment is bolstered by Kontar \& Brown's (2006) finding, using a comparison of the brightness of the primary source with that of the photospherically-backscattered albedo patch (Kontar et al. 2006), that the electron distribution at energies $E \lesssim 200 \mathrm{keV}$ in the 2005 January 17 event was also consistent with isotropy.

EPK was supported by a PPARC Advanced Fellowship and by a grant from the Royal Society; AGE was supported by NASA Grant NNG04G063G and by subcontract SA4878-26308 from the University of California, Berkeley. The overall effort has greatly benefited from support by a grant from the International Space Science Institute (ISSI) in Bern, Switzerland.

\section{REFERENCES}

Brown, J.C., Emslie, A.G., \& Kontar, E.P. 2003, ApJ, 595, L115

Brown, J.C., Emslie, A.G., Holman, G.D., JohnsKrull, C.M., Kontar, E.P., Lin, R.P., Massone, A.M., \& Piana, M. 2006, ApJ, 643, 523

Dennis, B.R. 1985, Sol. Phys., 118, 49

Haug, E. 1975, Z. Naturforsch, 30, 1099

Haug, E. 1989, A\&A, 218, 330

Haug, E. 1998, Sol. Phys., 178, 341

Heristchi, D. 1986, ApJ, 311, 474

Holman, G.D., Sui, L., Schwartz, R.A., \& Emslie, A.G. 2003, ApJ, 595, L97

Koch, H.W., \& Motz J.W., 1959, Phys. Rev., 31, 920

Kontar, E.P., \& Brown 2006, ApJ, 653, 149

Kontar, E.P., MacKinnon, A.L., Schwartz, R.A., and Brown, J.C., 2006, Astronomy \& Astrophysics, 446, 1157.

Kozlovsky, B., Murphy, R.J., \& Ramaty, R. 2002, ApJS, 141, 523

Li, P. 1995, ApJ, 443, 855

Piana, M. Massone, A. M., Kontar, E.P., Emslie, A.G., Brown, J.C., \& Schwartz, R.A. 2003, ApJ, 595, L127

Ramaty, R., Kozlovsky, B., \& Lingenfelter, R.E. 1979, ApJS, 40, 487
Schwartz, R.A., et al. 2002, Sol. Phys., 210, 165

Smith, D. M., Lin, R. P., Turin, P. et al., 2002, Solar Physics, 210, 33

Trottet, G., Vilmer, N., Barat, C., Benz, A., Magun, A., Kuznetsov, A., Sunyaev, R., \& Terekhov, O. 1998, A\&A, 334, 1099

Vestrand, W.T. 1988, Sol. Phys., 118, 95

This 2-column preprint was prepared with the AAS LATEX macros v5.2. 